\title{
Exploiting better motion cues for better action recognition
}

\author{
Usmain Ahmed, Jawad Khan
}

\section{INTRODUCTION}

Several recent studies on action recognition have emphasised the significance of including motion characteristics clearly in the video description. This work shows that properly partitioning visual motion into dominant and residual motions enhances action recognition algorithms greatly, both in terms of extracting space-time trajectories and computing descriptors. Then, using differential motion scalar variables, divergence, curl, and shear characteristics, we create a new motion descriptor, the DCS descriptor. It adds to the results by capturing additional information on local motion patterns. Finally, adopting the recently proposed VLAD coding technique in image retrieval improves action recognition significantly. On three difficult datasets, namely Hollywood 2, HMDB51, and Olympic Sports, our three additions are complementary and lead to beat all reported results by a large margin.

\section{REFERENCES}

[1] Gowda, S.N., 2017. Human activity recognition using combinatorial Deep Belief Networks. In Proceedings of the IEEE Conference on Computer Vision and Pattern Recognition Workshops (pp. 1-6).

[2] Wang, H. and Schmid, C., 2013. Action recognition with improved trajectories. In Proceedings of the IEEE international conference on computer vision (pp. 3551-3558).

[3] Jhuang, H., Gall, J., Zuffi, S., Schmid, C. and Black, M.J., 2013. Towards understanding action recognition. In Proceedings of the IEEE international conference on computer vision (pp. 3192-3199).

[4] J. Carreira and A. Zisserman. Quo vadis, action recognition? a new model and the kinetics dataset. In proceedings of the IEEE Conference on Computer Vision and Pattern Recognition, pages 6299-6308, 2017. [5] T. Cloete and C. Scheffer. Benchmarking of a full-body inertial motion capture system for clinical gait analysis. In 2008 30th Annual International Conference of the IEEE Engineering in Medicine and Biology Society, pages 4579-4582. IEEE, 2008.

[6] A. Colim, P. Carneiro, N. Costa, P. M. Arezes, and N. Sousa. Ergonomic assessment and workstation design in a furniture manufacturing industrya case study. In Occupational and Environmental Safety and Health, pages 409-417. Springer, 2019.

[7] J. Dai, K. He, and J. Sun. Convolutional feature masking for joint object and stuff segmentation. In Proceedings of the IEEE Conference on Computer Vision and Pattern Recognition, pages 3992-4000, 2015.

[8] Gowda, S.N. and Yuan, C., 2018, December. ColorNet: Investigating the importance of color spaces for image classification. In Asian Conference on Computer Vision (pp. 581-596). Springer, Cham.

[9] Y. Du, W. Wang, and L. Wang. Hierarchical recurrent neural network for skeleton based action recognition. In Proceedings of the IEEE conference on computer vision and pattern recognition, pages 1110-1118, 2015.

[10] X. Guo, J. Liu, and Y. Chen. Fitcoach: Virtual fitness coach empowered by wearable mobile devices. In IEEE INFOCOM 2017-IEEE Conference on Computer Communications, pages 1-9. IEEE, 2017.

[11] I. Habibie, W. Xu, D. Mehta, G. Pons-Moll, and C. Theobalt. In the wild human pose estimation using explicit $2 \mathrm{~d}$ features and intermediate $3 \mathrm{~d}$ representations. In Proceedings of the IEEE Conference on Computer Vision and Pattern Recognition, pages 10905-10914, 2019.

[12] Gowda, S.N., Rohrbach, M. and Sevilla-Lara, L., 2021, May. SMART Frame Selection for Action Recognition. In Proceedings of the AAAI Conference on Artificial Intelligence (Vol. 35, No. 2, pp. 1451-1459). 